\title{
Association of BEN Glycoprotein Expression with Climbing Fiber Axonogenesis in the Avian Cerebellum
}

\author{
Olivier Pourquié, Marc E. R. Hallonet, and Nicole M. Le Douarin \\ Institut d'Embryologie Cellulaire et Moléculaire du CNRS et du Collège de France, 94736 Nogent sur Marne Cedex \\ France
}

\begin{abstract}
In a previous study, we have identified an avian $100 \mathrm{kDa}$ membrane glycoprotein that we called BEN and demonstrated that it is transiently present in the CNS and PNS on the cell somas and axons of neurons that establish the peripheral neuronal circuitry. We report here that in the developing chick cerebellar system BEN is selectively expressed on fibers whose ingrowth and synaptogenesis pattern corresponds to that described for climbing fibers. We have constructed quail-chick chimeras in which the chick mesencephalon and anterior metencephaton were replaced by their quail counterparts, thus generating a cerebellum and mesencephalon exclusively composed of quail cells whereas the main nuclei emitting afferent fibers to the cerebellar cortex were of chick origin. Then, using species-specific monoclonal antibodies we were able to show in double staining experiments that BEN protein is specifically expressed on fibers arising from the inferior olivary nucleus. The spatiotemporal pattern of BEN expression on the climbing fibers leads us to propose that this molecule is associated with the growth of these fibers and with the establishment of synapses between them and the Purkinje cell dendritic tree.
\end{abstract}

We have recently described a novel membrane glycoprotein whose pattern of expression is developmentally regulated in the nervous system of a vian embryos (Pourquié et al., 1990). During ontogeny, expression of this protein is restricted to the period when ingrowing growth cones and axons actively establish the peripheral neuronal circuitry. The protein, known as BEN (MW 95-100 kDa), is recognized by a monoclonal antibody (anti$\mathrm{BEN} \mathrm{mAb}$ ) that was generated in mice against surface determinants of the epithelial component of the bursa of Fabricius (Pourquié et al., 1990). In the embryonic nervous system, this antibody recognizes several types of neurons whose axons proj-

\footnotetext{
Received June 28, 1991; revised Nov. 15, 1991; accepted Nov. 25, 1991

We are particularly indebted to Dr. S. Takagi (Department of Medecine, Kyoto Prefectural University) for the gift of the mAbs 39B11 and 37F5. We thank Jacqueline Giosué and Marie-Claude Birot for excellent histological assistance. We are also particularly grateful to Drs. Deborah Hall and Taube Rothman for their critical reading of the manuscript and to Bernard Henri, Yann Rantier, and Sophie Gournet for photography and artwork. Financial support was provided by the Centre National de la Recherche Scientifique (CNRS), the Fondation pour la Recherche Médicale Française, and the Ligue Française pour la Recerche contre le Cancer. O.P. is a recipient of a fellowship from the CNRS and M.E.R.H. is the recipient of an AMN fellowship from the University Paris XIII.

Correspondence should be addressed to Olivier Pourquié, Institut d'Embryologie, Cellulaire et Moleculaire, 49 bis avenue de la Belle Gabrielle, 94736 Nogent sur Marne Cedex, France.

Copyright (c) 1992 Society for Neuroscience $0270-6474 / 92 / 121548-10 \$ 05.00 / 0$
}

ect to the periphery. These include the motor neurons of the brain and spinal cord, sensory neurons of dorsal root ganglion (DRG), cranial ganglia, the sympathetic ganglia, and the neurons of the enteric nervous system. Downregulation of BEN first occurs in cell bodies, during the period of synaptogenesis, and subsequently spreads to axons and terminals. BEN is expressed at the surface of neurons as evidenced by staining of living cell suspensions, and this molecule bears the HNK-1 epitope (Pourquié et al., 1990), which is thought to be implicated in regulation of cell adhesion (Künemund et al., 1988). These observations have led to the hypothesis that BEN might play a role in the cell/cell or cell/matrix interactions that lead to the establishment of the peripheral axonal network.

$\mathrm{BEN}$ protein is also present in the brain and in the cerebellum, where its pattern of expression was found to be intriguing. The embryonic development of the cerebellum, well described by Ramon y Cajal (1911), has subsequently been characterized in several species, including birds (Ramon y Cajal, 1911; Mugnaini, 1969). Two afferent systems connect the cerebellar cortex to the rest of the brain: mossy and climbing fibers. Mossy fibers originate from various precerebellar nuclei, such as the pontine nuclei, as well as the reticular formation, the spinal cord, and various other sources (Eccles et al., 1967). These are myelinated fibers that make a peculiar type of synaptic contact with the axons of Golgi cells and dendrites of granular cells (glomeruli) in the internal granular layer. Climbing fibers, on the other hand, are considered as arising exclusively from the neurons of the inferior olivary nucleus. The behavior of growing climbing fibers, first described by Ramon y Cajal (1911), has been documented in various species by a number of workers (for a review, see Brodal and Kawamura, 1980; Mason, 1987). These fibers undergo a complex program of development. Initially they form a nest (" nid") around the Purkinje cells before climbing on apical dendrites to synapse on dendritic ramifications.

The present study was aimed at investigating the expression of BEN during the ontogeny of the avian cerebellum. The monoclonal antibody directed against the BEN glycoprotein (antiBEN mAb) was used on sections of chick brains at increasing developmental stages. BEN immunoreactivity was found to be strictly associated with fibers whose spatial and temporal developmental pattern corresponds to those of climbing fibers.

As opposed to mammals where the period of climbing fiber ingrowth takes place during the early postnatal period, this process occurs in the chick during the second half of incubation (8-21 d) when the animal is hardly accessible to experimental manipulations. The use of quail-chick chimeras (Le Douarin, 1982) seemed particularly appropriate to investigate whether 
the BEN-bearing fibers were extrinsic in origin to the cerebellum. By using the quail-chick chimera experimental paradigm, it was shown in this laboratory that the presumptive territory of the cerebellum corresponds to the caudal half of the mesencephalon and to the anterior metencephalon (Hallonet et al., 1990; M.E.R. Hallonet and N.M. Le Douarin, unpublished observations). Moreover, using the same technique, Tan and Le Douarin (1991) demonstrated that most of the inferior olivary nucleus, from which the climbing fibers originate, arises from the myelencephalon. It was then possible to construct chimeras in which the cerebellum would be quail and the climbing fibcrs chick. This was achieved by the substitution of the mesencephalon and rostral metencephalon of a chick embryo at the 12-somite stage by their counterpart coming from a stage-matched quail. This yielded chimeric animals whose cerebellum, optic tectum, and mesencephalic derivatives were entirely composed of quail cells whereas the afferent fibers to the cerebellar cortex originating from the inferior olivary nucleus (for the climbing fibers), the nuclei pontis lateralis and medialis, and the spinal cord (for the mossy fibers) were of chick origin. Chick fibers invading the cerebellum could then be evidenced by using a specific mAb directed against chick neuronal fibers (Takagi et al., 1988). This led to the demonstration that climbing fibers express transiently the BEN protein during their ascension to the Purkinje cell layer of the cerebellar cortex.

\section{Materials and Methods}

Embryos. Fertilized eggs from chick (Gallus gallus domesticus) and quail (Coturnix coturnix japonica) were obtained from commercial sources and were incubated in a rotary incubator at $37.5^{\circ} \mathrm{C}$. Stages of development are expressed in days of incubation and in number of somites for the early developmental stages. Ex means embryonic day $x$.

Surgery, quail-chick chimeras. Surgery was performed, as previously described (Le Douarin, 1982; Hallonet et al., 1990), on chick embryos at the 12-somite stage. Briefly, after removal of approximately $0.5 \mathrm{ml}$ of albumin from the egg, a window was opened in the shell, and following the surgical procedure the window was closed with adhesive tape and the operated eggs were reincubated at $37.5^{\circ} \mathrm{C}$ and $70 \%$ humidity. The transverse constrictions limiting the metencephalic vesicle from the myelencephalon caudally and the mesencephalon rostrally were used to define the limits of the operation. Isotopic and isochronic replacement of the chick mesencephalic vesicle and of the anterior part of the metencephalic vesicle by their quail counterparts was performed (see Fig. 6). Surgical dissociation of the neuroepithelium was done in both donor and recipient as described earlier (Hallonet et al., 1990). Operated embryos were incubated until E10 and E15. Only the chimeras presenting a strictly normal appearance both at the gross anatomy and histological levels were retained for analysis.

Antibodies and biochemistry. The anti-BEN mAb, obtained as previously described (Pourquié et al., 1990), is a mouse IgG1. mAbs 37F5 and $39 \mathrm{~B} 11$ are mouse antibodies. The supernatants were kindly provided by Dr. Takagi (Dept. of Medicine, Tokyo Prefectural University). They recognize antigens expressed by chick neuronal somas (37F5 mAb) and chick axons (39B11 mAb), respectively, at the exclusion of any antigen of quail origin (Takagi et al., 1988). A polyclonal antibody designated BENL2 was made in rabbit against BEN protein purified by means of an anti-BEN mAb affinity column. Affinity purification was carried out as described by Schneider et al. (1982) and Bastiani et al. (1987). Briefly, 100 E5 chick embryos were used per column, which yielded about $20 \mu \mathrm{g}$ of purified protein per column. The column eluate was precipitated in $20 \%$ trichloroacetic acid. For the first injection, the column eluate was run on a $7.5 \%$ SDS gel and blotted onto a Immobilon-P membrane with a semidry blotting apparatus (Ceralabo) as described previously (Dulac et al., 1988). The membrane was then stained with $0.1 \%$ Coomassie blue and washed for $1 \mathrm{hr}$ in water, and the band corresponding to the protein was cut out, dried, and placed into the spleen of a 4-month-old female rabbit anesthetized with pentobarbital ( $1 \mathrm{ml} / \mathrm{kg}$ ). Subsequent injections were performed at 3 week intervals with $20 \mu \mathrm{g}$ of protein (resuspended in PBS) electroeluted with a Bio-
Rad electroeluter according to the manufacturer's instructions. The second boost was injected into the ear vein, and the third one by injection into the spleen, under anesthesia. The rabbit was bled 10 and $14 \mathrm{~d}$ after the third injection, and the IgG fraction of serum was purified using the Bio-Rad IgG purification kit. Specificity of the polyclonal antibody to the purified protein was assessed on Western blot and on tissue extracts from chick and quail (see Fig. 5). Immunofluorescence using this antibody demonstrated colocalization with the anti-BEN mAb (see Fig. 7). The working dilution of the polyclonal antibody was $1 / 1000$ in PBS.

Histological and immunocytochemical procedures. For the ontogenetic study of BEN expression, the heads of young embryos (up to E10) or whole dissected brains from E11 to 6 months were fixed in Carnoy's solution and embedded in paraffin. Peroxidase immunocytochemistry was carried out on $5 \mu \mathrm{m}$ serial sections as described in Pourquié et al. (1990). Some chimeras were studied after staining sections with cresyl violet and according to the Feulgen-Rossenbeck procedure to identify the quail nuclear marker and thus to examine the extent of the territories deriving from the graft. Other chimeras were studied using immunocytochemical methods. Dissected brains of E10 and E15 chimeras were fixed overnight in 4\% paraformaldehyde in PBS, rinsed for $24 \mathrm{hr}$ in PBS and left overnight in PBS plus $20 \%$ sucrose, embedded in Tissue Tek, and frozen in liquid nitrogen. Analysis of chimerism was done by staining a series of three slides on which consecutive serial sections 15 $\mu \mathrm{m}$ thick were collected. The first slide was treated with $39 \mathrm{~B} 11 \mathrm{mAb}$ (which recognizes chick axons); the second, with 37F5 (which stains chick neuronal somas). Both antibodies were revealed with a peroxidasecoupled anti-mouse IgG antibody (immunostaining was carried out as for the paraffin sections). The third slide was double stained using the anti-BEN mAb directly coupled to fluorescein isothiocyanate (FITC) and $39 \mathrm{~B} 11 \mathrm{revealed}$ with a goat anti-mouse IgG coupled to Texas red (Southern Biotechnology Associates). All the sections were simultaneously stained with the Hoechst nuclear stain (33258) to visualize chick and quail nuclei. The slides were mounted in $90 \%$ glycerol in PBS, with paraphenylenediamine $(100 \mathrm{mg} / \mathrm{ml})$, and stored at $-20^{\circ} \mathrm{C}$. As a control, some of the slides previously double stained with anti-BEN and 39B11 $\mathrm{mAbs}$ were restained with polyclonal antibody BENL2 and 39B11 $\mathrm{mAb}$. One month after, fluorescence from the first series of staining had vanished. These sections were then treated first with a donkey anti-goat Ig antibody coupled to rhodamine isothiocyanate (Nordic) to show the 39B11 reactivity as a tertiary antibody. Subsequently, BENL2 was applied and revealed with a goat anti-rabbit antibody coupled to FITC (Nordic).

\section{Results}

Ontogenetic emergence of BEN glycoprotein in the chick inferior olivary nucleus and in precerebellar nuclei

BEN expression was first detected at E9, in the inferior olivary nucleus, soon after the cells had migrated from the rhombic lip (E7) and settled in the ventral part of the rhombencephalon (Harkmark, 1954; Tan and Le Douarin, 1991). Strong expression of the protein was observed in the inferior olivary nucleus during late embryonic development and in early posthatched chicks, but immunoreactivity totally disappeared in the adult. At E12, immunoreactive fibers emanating from the inferior olivary nucleus were observed crossing the floor plate before changing their direction of growth to the contralateral side. Bourrat and Sotelo (1988) have described a similar situation regarding the direction of projections from the olivary nucleus in the rat. In the chick inferior olivary nucleus, immunoreactivity was essentially present on neuronal cell bodies (Fig. 1) but was also observed on the dense meshwork of fibers in which the neurons were embedded.

Immunoreactivity was totally absent on the main precerebellar nuclei known to project to the cerebellar cortex regardless of stage (E5 to 6 months posthatching), that is, the nuclei pontis lateralis and medialis (Brodal et al., 1952) or the reticular formation. BEN immunoreactivity was never found on the spinocerebellar tracts of the spinal cord. 


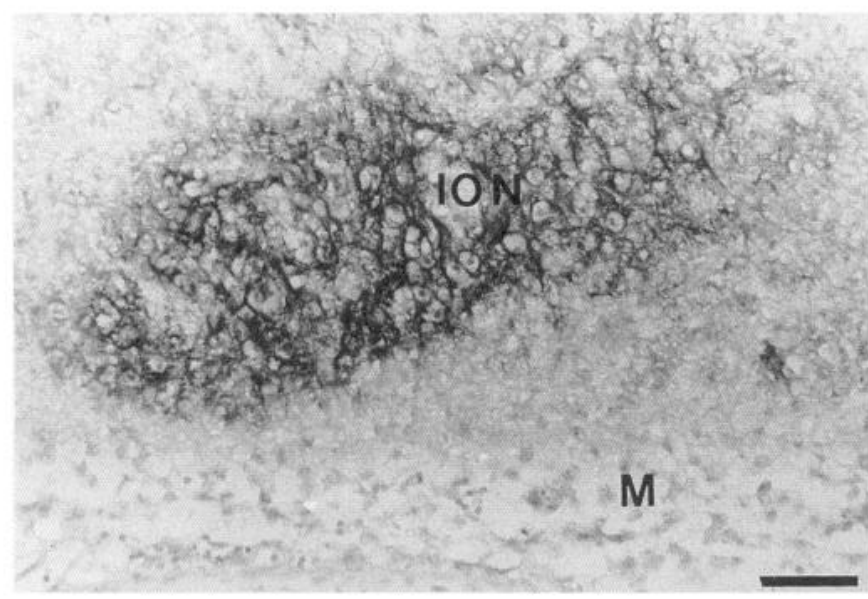

Figure 1. Immunoperoxidase staining with anti-BEN mAb of a transverse section of $\mathrm{E} 9$ chick brain, showing reactive neurons in the inferior olivary nucleus. Medial is to the left. $M$, meninges; $I O N$, inferior olivary nucleus. Scale bar, $130 \mu \mathrm{m}$.

\section{$B E N$ expression in the cerebellum during development}

$E 9$. The granular layer had almost completely covered the cerebellar plates at this stage, and Purkinje neurons had completed their migration from the underlying ventricular epithelium (Hallonet et al., 1990). BEN immunoreactivity could not be detected in the cerebellum. One day later, however, BEN expression was observed on fibers in the cerebellar peduncles as well as within the presumptive white matter of the cerebellum itself. At this stage, immunostaining was irregularly distributed in the zone located just beneath the external granular layer, where Purkinje cells were still randomly arranged.

$E 12$ and E13. Immunoreactivity was more intense than at E10 and continued to be observed on fibers of the cerebellar peduncles as well as on a meshwork of fibers in the future white matter. Maximum staining intensity could be discerned within discrete zones of the Purkinje cell layer, which was still multicellular at this stage.

$E 15$. The different components of the mature cerebellum were clearly recognizable, and anti-BEN immunoreactivity, which had increased in intensity, was particularly conspicuous on fibers in the cerebellar peduncles and white matter and was distributed in patches within the Purkinje cell layer (Figs. $2 A, D ; 3 A$ ). These patches were arranged in sagitally oriented stripes (Fig. 4), evoking the functional arrangement of olivocerebellar projections (Groenenwegen and Voogd, 1977). This periodic arrangement of immunoreactivity observed at a given time was not uniformly distributed throughout the cerebellum possibly because of the dynamic aspect of expression of this protein (Pourquie et al., 1990), and because all parts of the cerebellum do not develop isochronically. At E15, stained fibers in the white matter tracts had reached the internal granular layer where they ramified extensively to form the plexus that contacts the lower part of the Purkinje cell somas (Fig. 3A). This plexus may correspond to the so-called lamina dissecans, a transient fiber-rich zone underlying the Purkinje cell layer described in mammals (Rakic and Sidman, 1970; Marin-Padilla, 1985). The Purkinje cells contacted by this plexus of fibers were aligned at this stage, although they were not yet perfectly polarized.

E18. At this stage, immunoreactivity had reached its peak in the cerebellum. Strongly stained fibers were seen in the white matter, and those entering the internal granular layer followed a sinuous trajectory (Fig. $2 B, E$ ). Maturation of the cerebellar folia proceeded along a proximodistal gradient. In sagittal and parasagittal sections of the cerebellum, the relationships between the endings of climbing fibers and Purkinje cell bodies exhibited progressive stages of maturation: the supracellular stage ("en capuchon" according to Ramon y Cajal) was seen more proximally (Fig. $3 \mathrm{C}$ ), while the nest stage ("en nid" according to Ramon y Cajal) was more distal (Fig. $3 B$ ).

E19. Anti-BEN mAb labeling was no longer observed in fibers surrounding Purkinje cells. Immunoreactivity was still apparent in certain cerebellar areas where it was localized on the top of the Purkinje cell somas. In the molecular layer, there was conspicuous and diffuse staining associated with the terminations of climbing fibers when they made contacts with the dendritic tree of Purkinje cells.

Posthatched chick. The intensity of BEN immunoreactivity was much lower throughout the cerebellum (Figs. $2 C, F ; 3 D$ ). The strongest staining was observed in the molecular layer, but weakly stained fibers were also present within the white matter and internal granular layer. In the adult animal, only faint reactivity could be detected in the molecular layer.

\section{Specific labeling of cerebellar afferent projections in quail- chick chimeras}

Implantation of the mesencephalon and anterior metencephalon from quail to stage-matched chick embryos was performed as indicated on Figure $6 \mathrm{~A}$. Previous studies from our laboratory (Hallonet et al., 1990; Tan and Le Douarin, 1991; Hallonet and Le Douarin, unpublished observations) have shown that the entire presumptive territory of the cerebellum is included in this brain area at the exclusion of most of the inferior olivary nucleus originating from the myelencephalon. Six operated embryos were observed at E10 or E15. Three of them were treated to evidence the quail nuclear marker by using Feulgen-Rossenbeck and cres$\mathrm{yl}$ violet staining procedures. It appeared that, as expected, the cerebellum, optic tectum, and the other mesencephalic derivatives were entirely composed of quail cells whereas the inferior olivary nucleus neurons appeared to be essentially of chick origin. Nerve fibers invading the quail cerebellum and originating from the chick territories could be identified by using the 39B11 $\mathrm{mAb}$, which recognizes chick neuronal fibers, while BEN glycoprotein was detected on the same specimens by double staining with either anti-BEN mAb or BENL2, a polyclonal antibody raised against immunopurified $B E N$ protein (see Materials and Methods). BENL2 antibody recognizes, in Western blot, the same component as anti-BEN mAb (Fig. 5), and its pattern of immunoreactivity is strictly similar with anti-BEN mAb (see Fig. 7). 39B $11 \mathrm{mAb}$ detects exclusively afferent fibers of chick origin in the quail cerebellum of these chimeras. Analysis of chimerism was carried out by staining groups of three consecutive slides prepared as indicated in Materials and Methods (Fig. 6). Observation of 37F5 mAb-stained sections in which exclusively somas of chick neurons were immunoreactive confirmed that the cerebellum was entirely composed of quail cells (Fig. 6B). This was further substantiated by the use of the Hoechst nuclear reagent (Fig. 6C).

E10 chimeras. Strongly immunoreactive fibers were detected with $39 \mathrm{~B} 11 \mathrm{mAb}$ in the cerebellar peduncles, in the prospective white matter (Fig. $7 E$ ), and in patches located just beneath the 

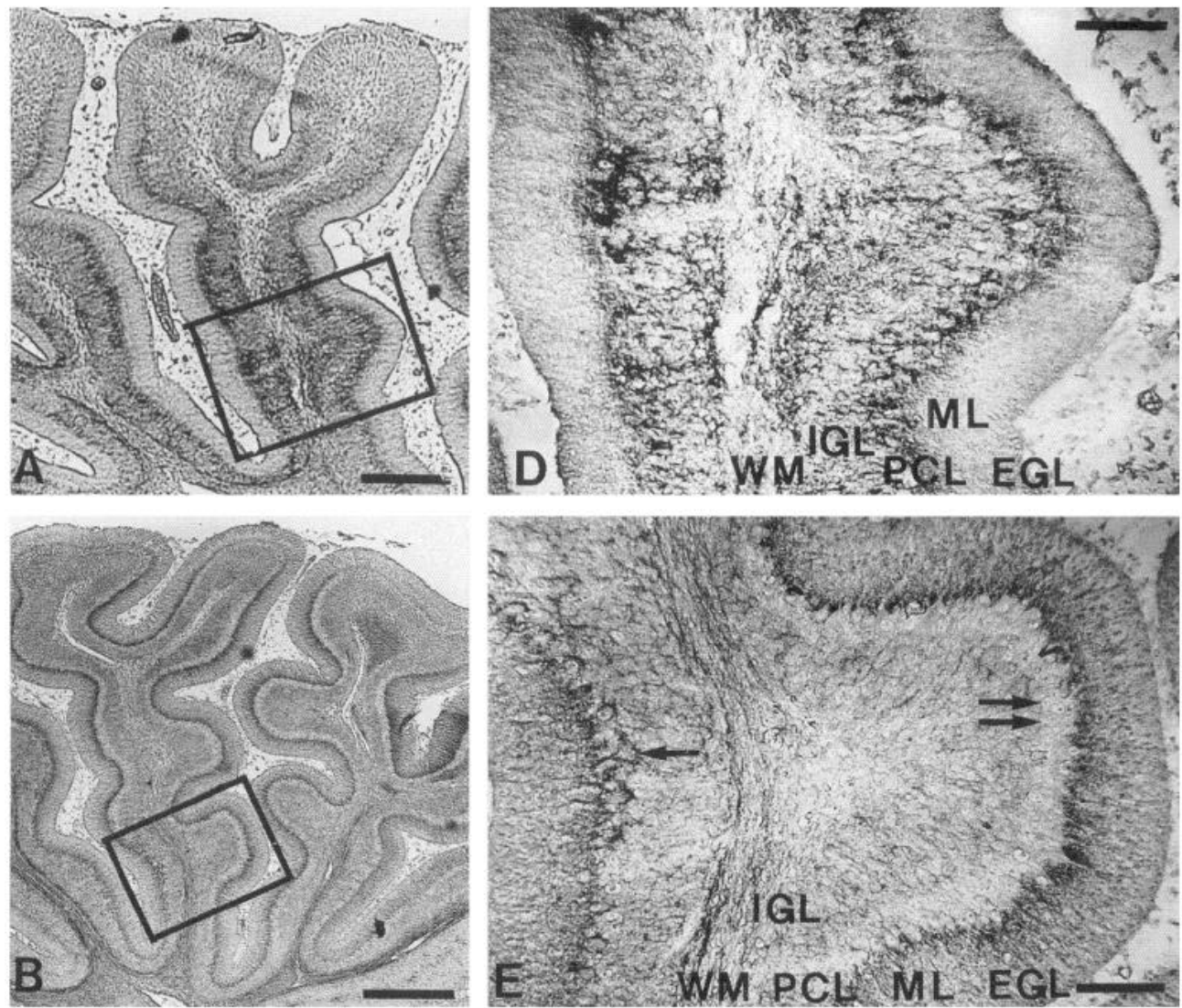

Figure 2. Sagittal sections of late developmental stages from E15 to postnatal day 2 (P2) of the chick cerebellum immunostained with anti-BEN mAb. Right column $(D-F)$ corresponds to higher magnification of the left column boxes $(A-C)$. $A$ and $D$ show an E15 cerebellum. In $D$ can be observed fibers coming from the white matter $(W M)$, crossing the internal granular layer $(I G L)$, and invading the Purkinje cell layer $(P C L)$. The molecular layer $(M L)$ and the external granular layer $(E G L)$ are totally devoid of immunoreactivity. $B$ and $E$ represent an E18 cerebellum. In $E$, the reactivity is still present in the white matter and the internal granular layer and is particularly strong in the Purkinje cell layer around the cell bodies and on the top of Purkinje cell somas. This presumably corresponds to climbing fibers nest stage (single arrow) and to more mature supracellular stage (double arrow). No reactivity is present in the molecular layer and in the external granular layer. $C$ and $F$, P2 cerebellum. In $F$, lower reactivity is detected in the white matter and the internal granular layer. At this stage, the maximal immunoreactivity appears to be in the molecular layer, where the climbing fibers are now ascending Purkinje cell dendrites. Scale bars: $A, 200$ $\mu \mathrm{m} ; B$ and $C, 330 \mu \mathrm{m} ; D$ and $E, 70 \mu \mathrm{m}$; $F, 100 \mu \mathrm{m}$

external granular layer (Fig. 6D). Expression of BEN protein could be detected with anti-BEN mAb and BENL2 on virtually all the 39B11 positive fibers (Fig. 7); however, since BEN expression was weak at that stage, it was not possible to ascertain whether all of the 39B11-positive fibers were double labeled.

E15 chimeras. BEN reactive fibers were detected in tracts within the white matter, surrounding Purkinje cells as well as on top of the Purkinje cell somas as described in the ontogenetic study carried out in normal chicks (Fig. 7). In the chimeras, all the BEN-positive fibers were $39 \mathrm{~B} 11$ positive. In some cases, 39B11-positive/BEN-negative fibers could be observed especially in the molecular layer. These very likely correspond to the final stage of climbing fiber maturation, when BEN immunoreactivity has considerably decreased.

\section{Discussion}

$B E N$ glycoprotein is expressed on climbing fibers in the chick cerebellum

The distribution pattern of BEN expression observed in the cerebellum corresponds to that expected of climbing fibers. There are several reasons why this appears to be so. First, the neurons in the inferior olivary nucleus are strongly immunoreactive during climbing fiber axonogenesis. Second, the growth pattern of the BEN-positive fibers and their relationships with Purkinje cells correlate well with the known maturation stages of the climbing fibers as they were described by Ramon y Cajal and several authors since.

As suggested by Altman and Bayer (1978) and by Bourrat 

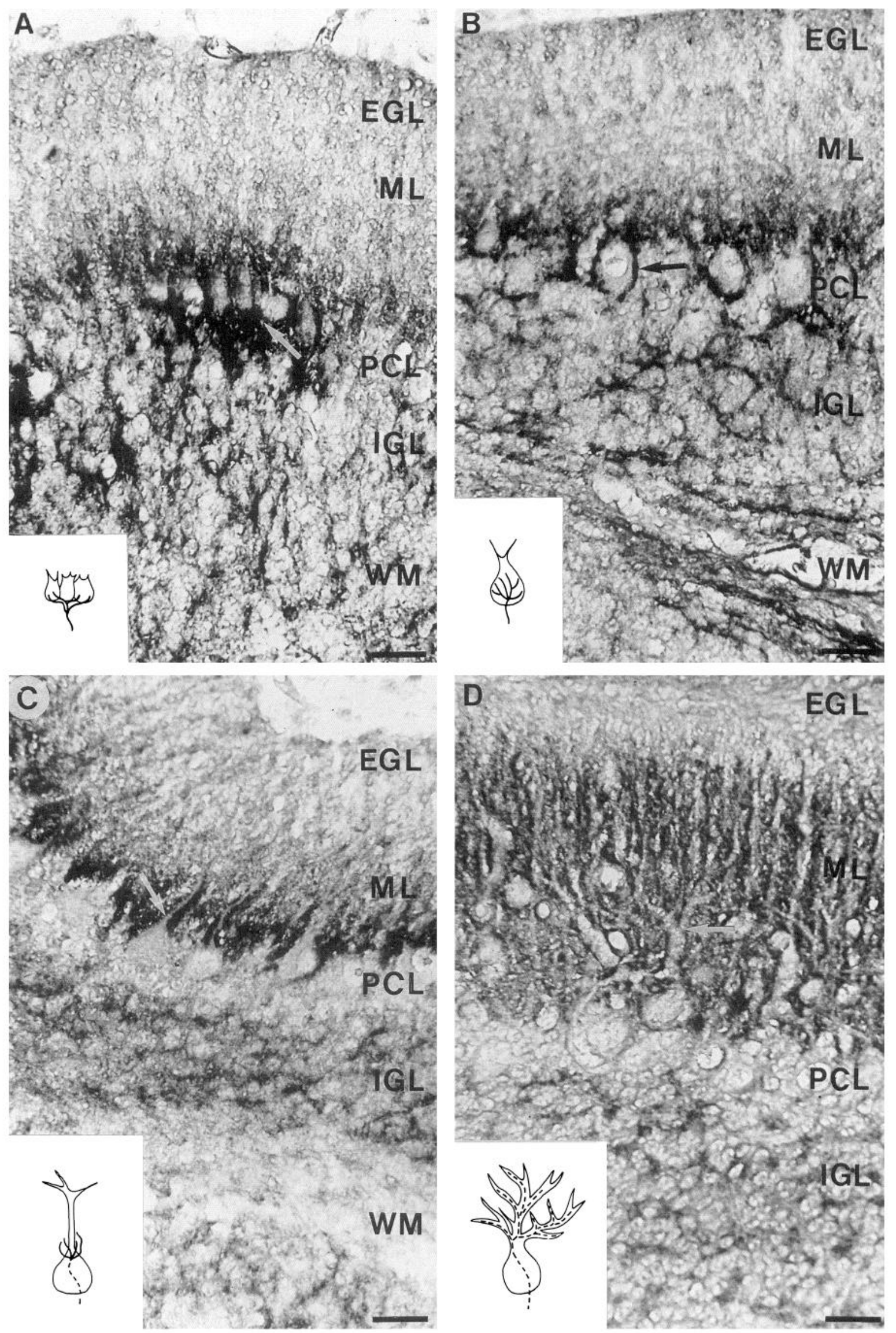


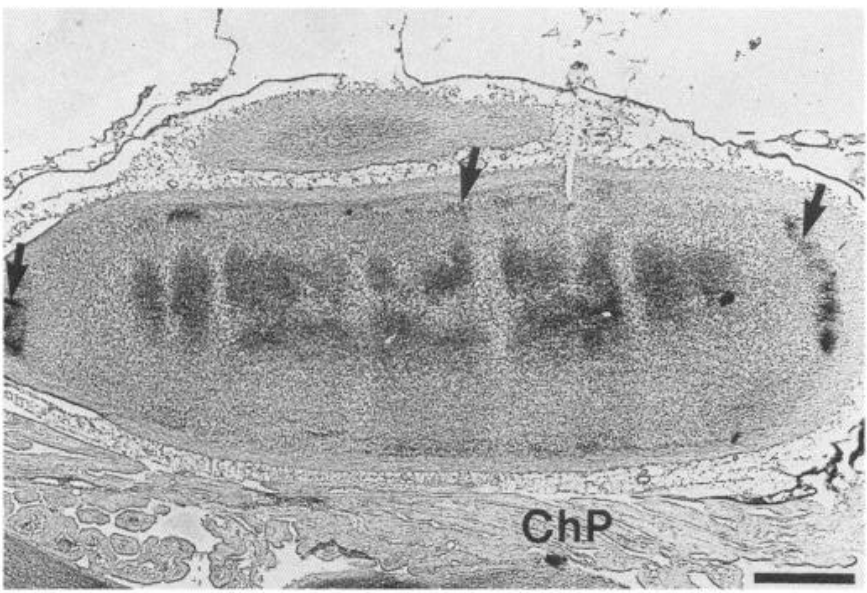

Figure 4. Transverse section of E15 folium X (nodulus) immunostained with anti-BEN mAb. A parasagittal arrangement of immunoreactivity in the internal granular layer is conspicuous. Arrows point to the Purkinje cell layer. $C h P$, choroid plexus. Scale bar, $250 \mu \mathrm{m}$.

and Sotelo (1988), the arrival of the first fiber from the inferior olivary nucleus is a precocious event. Altman and Bayer (1978) even suggested that the external granular layer spreads over a fibrous layer that could be composed of climbing fiber terminations. Similarly, Mason et al. (1990) proposed that climbing fibers could provide a migratory substrate for Purkinje cells when they migrate from the subventricular zone. Our findings are in agreement with the described timing since we detect the first immunoreactive fibers at E10 in the cerebellar peduncles, the prospective white matter (Fig. $7 E, F$ ), and as discrete patches in the area located beneath the external granular layer (Fig. 6D) just at the time when the external granular layer is completing its spreading over the cerebellar plate. Moreover, at this stage and in this region, no structure known to project to the cerebellum, other than the inferior olivary nucleus, does express the BEN antigen.

By E15, the cerebellar cortical layers are just becoming recognizable and immunoreactive fibers are observed crossing the internal granular layer and forming an extensive plexus beneath and surrounding the lower part of the Purkinje cell body as described in the mouse (Mason et al., 1990) when Purkinje cell alignment takes place. At this stage, immunoreactivity is maximal in the Purkinje cell layer and a sagittal arrangement of BEN-positive stripes can be detected (Fig. 4), as described for the pattern of the olivary projections in the rat (Groenenwegen and Voogd, 1977; Sotelo et al., 1984). In the chick, such a sagittal organization has also been described in the cerebellum (Feirabend, 1990).

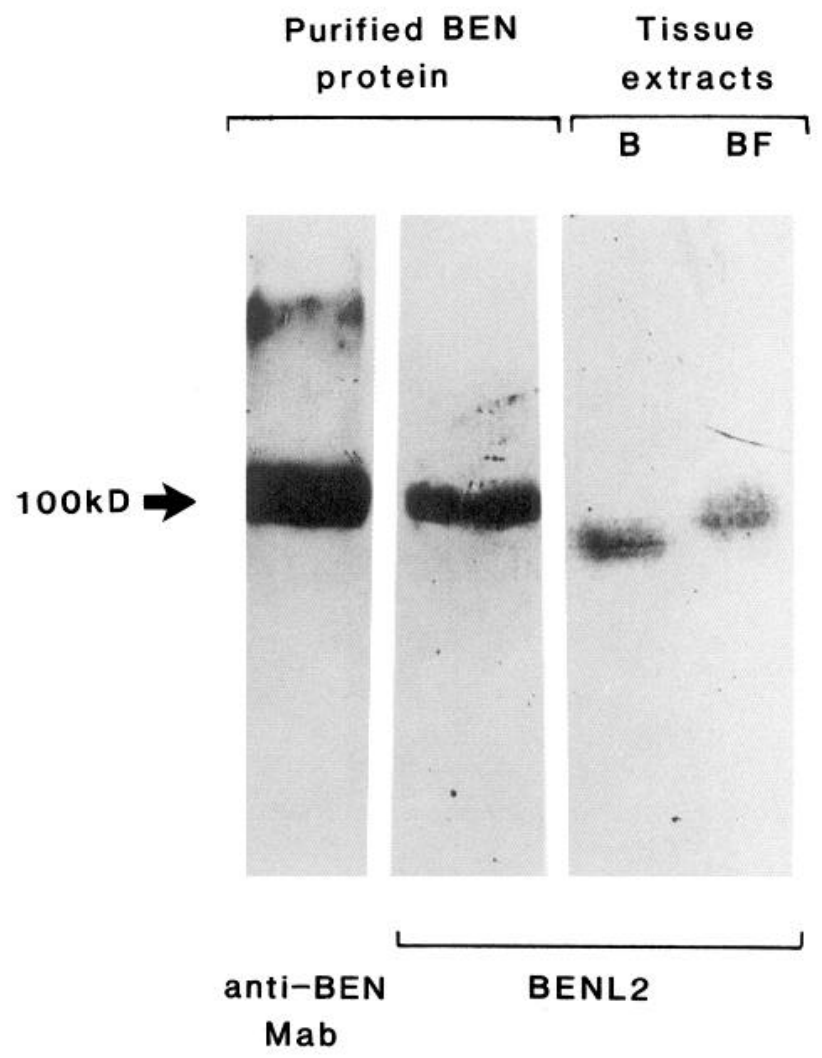

Figure 5. Western blot comparison of anti-BEN mAb and BENL2 polyclonal antibody reactivities after electrophoresis under nonreducing conditions. $B E N L 2$ antibody recognizes the BEN protein purified from E5 chick embryos as does anti-BEN mAb. In tissue extracts of brain $(B)$ and of bursa of Fabricius $(B F), B E N L 2$ recognizes bands of approximately 95 and of $100 \mathrm{kDa}$, respectively, as previously reported for anti-BEN mAb (Pourquié et al., 1990).

By E18, immunoreactive fibers enclose Purkinje cell somas, as in the "nid" or nest stage first described by Ramon y Cajal (Fig. $3 B$ ). In more mature parts of the cerebellum, maximum reactivity appears to be relocated at the top of the Purkinje cell somas surrounding the base of the apical dendrite, as in the "capuchon" stage (Fig. 3C), when the fibers ascend Purkinje cell dendrites. This is in agreement with the observations of Mugnaini (1969), who detected the first growing terminal arborizations of climbing fibers in the chick from E18 onward by electron microscopy.

The chimera experiments demonstrate that, in the cerebellum, BEN is exclusively expressed on afferent nerve fibers. When quail mesencephalon and rostral metencephalon were grafted into a chick, the whole cerebellum was of quail origin as shown

Figure 3. High-magnification photographs of sagittal sections of chick cerebellum from E15 to P2 reacted with anti-BEN mAb. These pictures illustrate evolution of the BEN expression in the cerebellar cortex. A schematic drawing of a tentative interpretation of the staining is illustrated in the lower left corner, where relationships between climbing fibers and Purkinje cells are evidenced. Stained fibers are shown by a solid line; disappearance of the staining is illustrated by a broken line. $A, \mathrm{E} 15$. The BEN-positive fibers coming out of the white matter (WM) are arranged as a plexus contacting the bottom of the Purkinje cell somas (arrow), which are still imperfectly polarized at that stage. B, E18. BEN-positive fibers are completely surrounding Purkinje cell somas (arrow) as in the nest stage described by Ramon y Cajal for the maturation of climbing fibers. $C$, E18. In more mature parts of the cerebellum, the staining has disappeared from the cell body and is now restricted to the bottom of the emerging dendrite (arrow) of the Purkinje cell, as in the supracellular stage of Ramon y Cajal. $D$, P2. Reactivity has diminished and is essentially present in the molecular layer where the Purkinje cell dendrite is now well ramified (arrow). This phase corresponds to the terminal phase of maturation of the climbing fibers, that is, the ascension of the Purkinje cell dendrite. $E G L$, external granular layer; $I G L$, internal granular layer; $M L$, molecular layer; $P C L$, Purkinje cell layer; $W M$, white matter. Scale bars, $20 \mu \mathrm{m}$. 


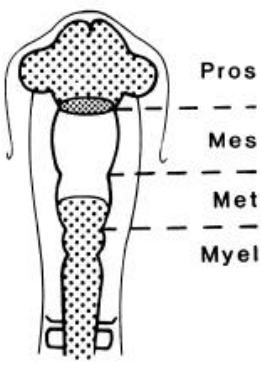

QUAIL

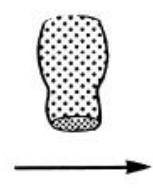

A

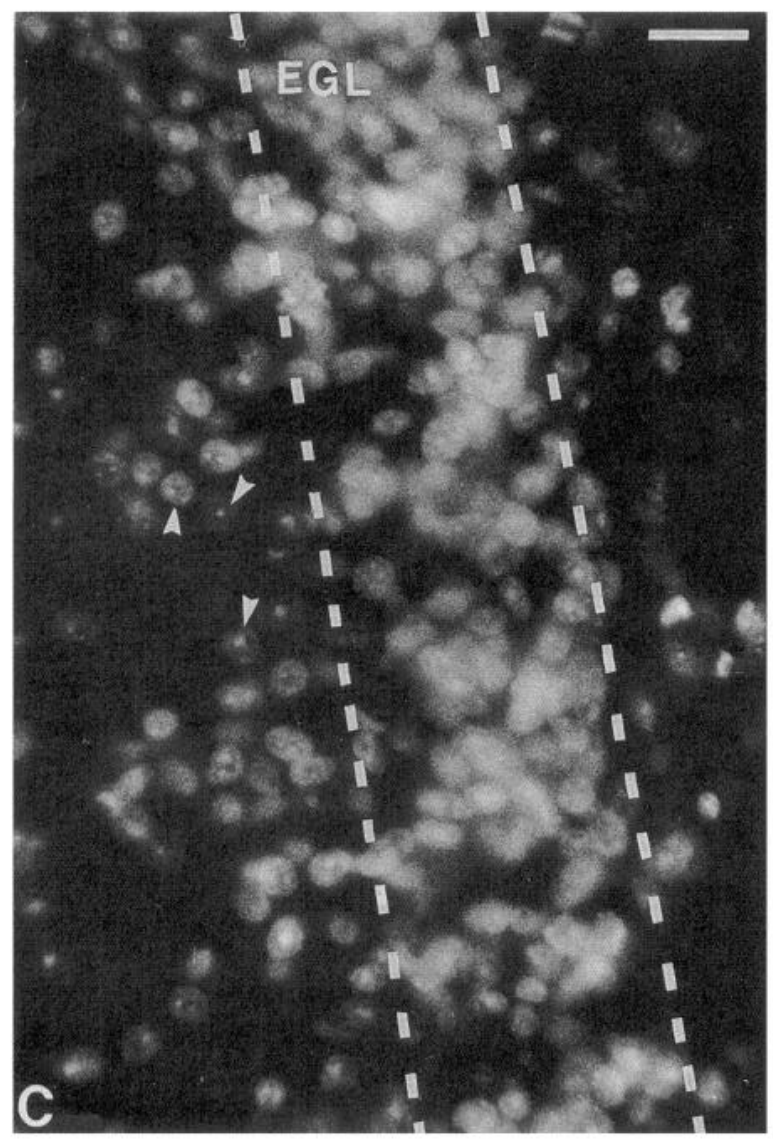

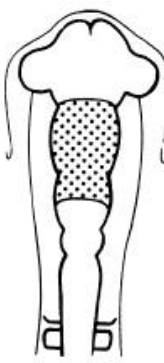

CHICK
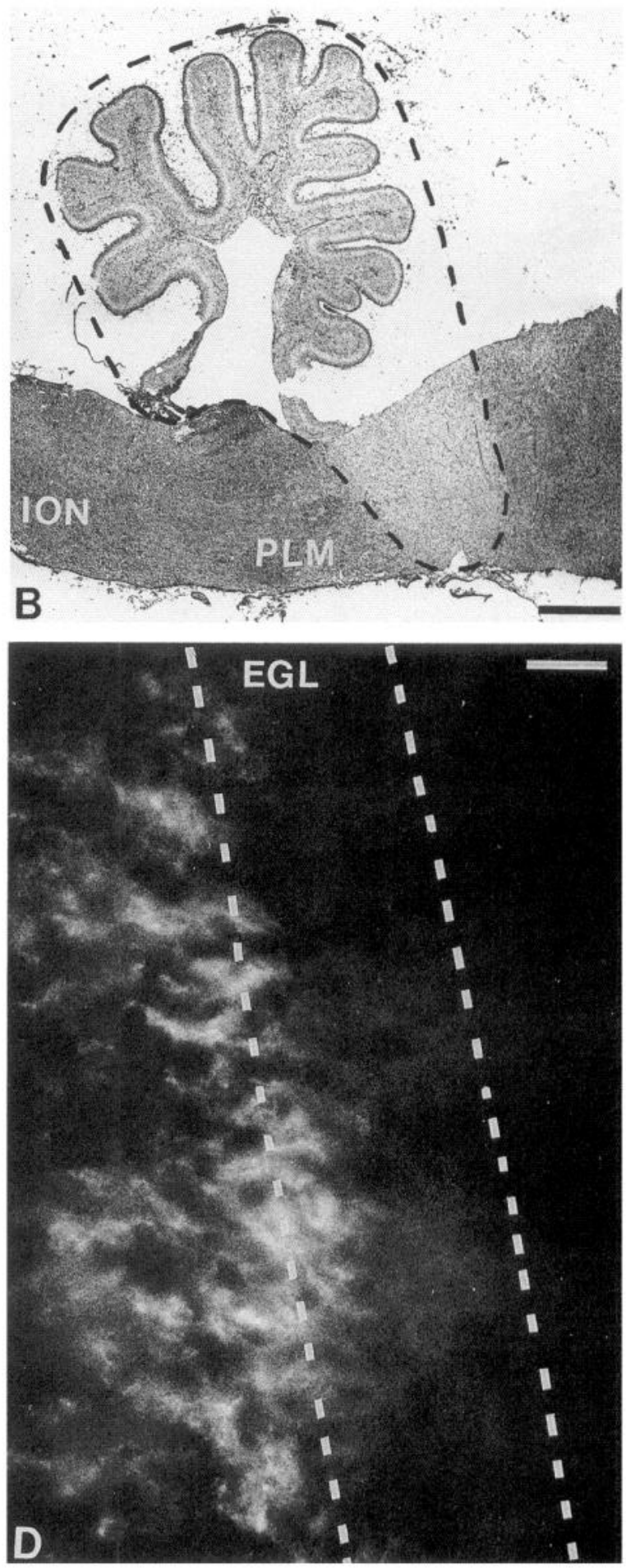

Figure 6. A, Schematic representation of the isotopic and isochronic transplantation of the quail mesencephalon and anterior metencephalon in a chick performed at the 12-somite stage. Pros, prosencephalic vesicle; Mes, mesencephalic vesicle; $M e t$, metencephalic vesicle; Myel, myelencephalic vesicle. $B$, Sagittal section of the brain of an E15 chimera. Immunoperoxidase staining with 37F5 chick cell body-specific mAb. The grafted area appears unreactive and is surrounded with a broken line. The punctate staining observed in the grafted area is probably due to chick endothelial cells that have migrated in the grafted territory. The emplacement of the inferior olivary nucleus (ION; source of climbing fibers) and of the pontis lateralis and medialis nuclei (PLM; source of pontine fibers) has been indicated to show that they are located in the chick area. $C$, Sagittal section of the cerebellum of an E10 chimera reacted with Hoechst nuclear stain showing the characteristic quail nuclei (arrowheads). The external granular layer $(E G L)$ is delimited by broken lines. $D$, Staining of the same view with 39B11 chick fiber-specific mAb showing their location beneath the $E G L$ in the prospective Purkinje cell zone. Scale bars: $B, 1 \mathrm{~mm} ; C$ and $D, 60 \mu \mathrm{m}$. 

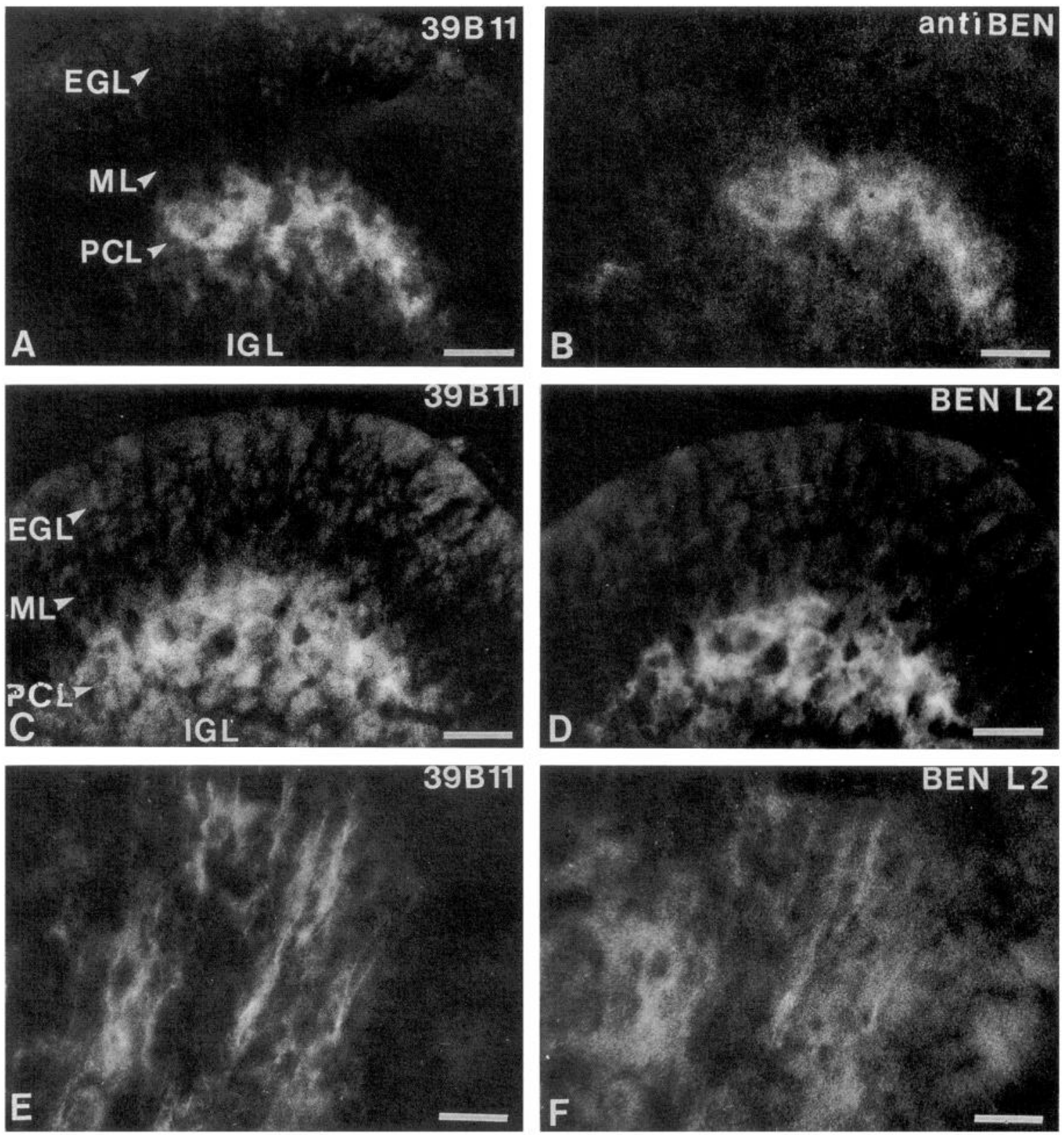

Figure 7. Double staining experiments on sagittal sections of cerebellum of E15 $(A-D)$ and $\mathrm{E} 10(E, F)$ quail-chick chimeras illustrating the identity of the fibers recognized by BEN mAb, BENL2 polyclonal antibody, and 39B11. $A$ and $B$ correspond to the same field. $A$ corresponds to the $39 \mathrm{~B} 11$ reactivity characterizing the chick neuronal fibers. $B$ illustrates anti-BEN mAb staining using the antibody directly coupled to FITC. $A$ and $B$ show the identity of the BEN- and 39B11-positive fibers observed in the Purkinje cell layer. $C$ and $D$ correspond to the same view of a slide restained with BENL2 antibody as described in Materials and Methods. $C, 39 \mathrm{~B} 11 \mathrm{mAb} . D$, BENL2 polyclonal antibody. The reactivity observed is identical and corresponds to that expected from the ontogenic study. $E$ and $F$ show the same field in the white matter of the cerebellum of an El0 chimera stained with mAb 39B11 $(E)$ and with BENL2 $(F)$. EGL, external granular layer; $M L$, molecular layer; $P C L$, Purkinje cell layer; $I G L$, internal granular layer. Scale bars, $40 \mu \mathrm{m}$.

by means of chick-specific antibodies and of Hoechst and Feulgen-Rossenbeck nuclear stains (Fig. 6). Only chick afferent fibers whose origins lie posterior to the anterior metencephalon or anterior to the mesencephalon such as the climbing fibers from the inferior olivary nucleus, and the fibers from the nuclei pontis lateralis and medialis and spinal cord, are recognized by the chick fiber-specific antibody 39B11. Double staining with 39B11 and anti-BEN mAbs or BENL2 polyclonal antibody revealed an almost perfect overlap between the two immunoreactivities
(Fig. 7). This demonstrates therefore that BEN immunoreactivity is present on afferent fibers.

In chick as in mammals, two main afferent fiber systems have been described in the cerebellum: the climbing and the mossy fibers (Whitlock, 1952; Harkmark, 1956; Vogt-Nilsen, 1959; Mugnaini and Forstronen, 1967; Mugnaini, 1969). The mossy fiber input is mostly derived from the pontine nuclei and various other sources such as the reticular formation and the spinal cord (Eccles et al., 1967). We were not able to detect any BEN reac- 
tivity during ontogeny either on the avian homolog of the pontine nuclei (Brodal et al., 1952), the nuclei pontis lateralis and medialis, and on the spinocerebellar tracts originating from the spinal cord. The sequence of reactivity observed in the cerebellum does not evoke the steps previously described for mossy fiber maturation (Mugnaini, 1969; Mason and Gregory, 1984; Mason et al., 1990). Namely, no immunoreactivity was ever observed at any stage in the internal granular layer that could be related to mossy fiber terminals. Moreover, in the rat, it was shown that mossy fibers reach the cerebellum much later than the climbing fibers (Altman and Bayer, 1978). Since the relative timing of these processes is very likely to be identical in the avian class, the BEN-positive fibcrs invading the ccrcbellum as early as E10 cannot be considered of the mossy type.

\section{$B E N$ expression is associated with axonogenesis and synaptogenesis}

We have previously reported the association of BEN expression with axonogenesis of peripherally projecting neurons (motoneurons, DRG neurons, parasympathetic and sympathetic neurons). In the olivocerebellar system, BEN expression is also associated with the phase of axonal elongation. First, it appears on olivary neurons very early in development (E8-E9), when the cerebellum is still rudimentary; then, as early as E10, reactive fibers are detected in the cerebellar peduncles, in the white matter, and in the Purkinje cell area. Therefore, it can be concluded that climbing fibers are immunoreactive over their entire length at these early stages, that is, from the inferior olivary nucleus to the Purkinje cell layer. The situation begins to change at E15 when at the "nid" and "capuchon" stages, immunoreactivity decreases in the internal granular layer and in the white matter while remaining strong around the Purkinje cells and at the base of the apical dendrites. The next step is illustrated in the young chick, where only a diffuse and faint reactivity is present in the molecular layer where climbing fiber terminations are now contacting the Purkinje cell dendrites. At this stage, BEN expression is barely detectable in the internal granular layer or the white matter. Therefore, as reported for motor and sensory neurons, expression of BEN protein stops around the time of synaptogenesis and its disappearance proceeds in a centrifugal way from the cell body to the axon extremity. This situation for the cerebellum is schematized in Figure 3. Like motor and sensory neurons, the downregulation of BEN on climbing fibers is concomitant with (Macklin and Weill, 1985) and might be related to the myelination process, a problem that we are presently investigating. It is worth noticing that strong anti-BEN immunoreactivity can be detected in suspensions of living neurons either from sympathetic ganglia or from ventral medulla oblongata (including the olivary nucleus), showing that this protein is expressed on the cell surface.

The already described distribution of various other cell surface glycoproteins during chick or mouse cerebellar development was compared to that observed for BEN. Ll and Ng-CAM, strongly expressed by the granule cell axons and by the fibers of the white matter tracts (Daniloff et al., 1986; Persohn and Schachner, 1987), were proposed to play a role in fasciculation events. $\mathbf{L 1}$, which is not expressed by stellate and basket cells, seems to be specific for certain cerebellar neuronal subpopulations (Persohn and Schachner, 1987). The different isoforms of N-CAM are also transiently present on certain cerebellar populations of neurons and glial cells (Persohn and Schachncr, 1987; Hekmat et al., 1990), and neurofascin (Rathjen et al., 1987) is present on parallel fibers and on fibers of the white matter tracts. All the other proteins present on afferent fibers are also expressed on various other cell types in the cerebellum. It is remarkable that BEN is, so far, the only surface molecule specific for climbing fibers in the cerebellar system.

This specificity raises the problem of the function of this protein, which bears the HNK-1 epitope (Pourquié et al., 1990), suggesting that it might be implicated in adhesion phenomena (Künemund et al., 1988). On the basis of its temporal pattern of expression, one can assume that BEN glycoprotein is implicated in both axon elongation and synaptogenesis. Cessation of BEN synthesis would in this view be triggered by a signal arising from Purkinje cclls once synaptogencsis of Purkinje cells on their dendritic tree is completed.

It is notable that this protein is expressed only on one of the two afferent cerebellar systems. The mossy fibers have their source in areas located at some distance from the cerebellar cortex. Like the climbing fibers, they are myelinated. However, the two types of fibers differ in the timing of their growth, which occurs later for the mossy fibers (Altman and Bayer, 1978), and in their mode of termination, which occurs within the glomeruli of the internal granular layer for the mossy fibers and within synapses on the Purkinje cell dendritic tree for the climbing fibers. If adhesion molecules are important for the progression of incoming fibers as they navigate to their targets within the developing brain, one can imagine that mossy and climbing fibers use different recognition systems.

If BEN antigen expression is associated with an active remodcling of synaptic contacts, its ncarly continuous presence in the enteric nervous system would imply that not only the absence of myelination (see above) but also a high level of plasticity exists throughout life in the intrinsic innervation of the gut. These questions need to be further investigated before receiving a satisfactory answer. In any case, an important step toward the understanding of the potential role of BEN protein in the complex interactions leading to the establishment of connectivity in the brain would be to know the structure of the gene encoding this molecule and the regulations to which it is subjected during neurogenesis.

\section{References}

Altman J, Bayer S (1978) Prenatal development of the cerebellar system in the rat. II. Cytogenesis and histogenesis of the inferior olive, pontine gray, and the precerebellar reticular nuclei. J Comp Neurol 179:49-76.

Bastiani MJ, Harrelson AL, Snow PM, Goodman CS (1987) Expression of fasciclin I and II glycoproteins on subsets of axons pathways during neuronal development in the grasshopper. Cell 48:745-755.

Bourrat F, Sotelo C (1988) Migratory pathways and neuritic differentiation of inferior olivary neurons in the rat embryo. Axonal tracing study using the in vitro slab technique. Dev Brain Res 39:19-37.

Brodal A, Kawamura K (1980) The olivocerebellar projection. Adv Anat Embryol Cell Biol 64:1-140.

Brodal A, Kristiansen K, Jansen J (1952) Experimental demonstration of a pontine homolog in birds. J Comp Neurol 97:23-69.

Daniloff JK, Chuong CM, Levi G, Edelman G (1986) Differential distribution of cell adhesion molecules during histogenesis of the chick nervous system. J Neurosci 6:739-758.

Dulac C, Cameron-Curry P, Ziller C, Le Douarin NM (1988) A surface protein expressed by avian myelinating and non myelinating Schwann cells but not by satellite or enteric glial cells. Neuron 1:211-220.

Eccles JC, Ito M, Szentagothai J (1967) The cerebellum as a neuronal machine. Berlin: Springer.

Feirabend HKP (1990) Development of the longitudinal patterns in the cerebellum of the chicken (Gallus domesticus): a cytoarchitectural 
study on the genesis of cerebellar modules. Eur J Morphol 28:169223.

Groenenwegen HJ, Voogd J (1977) The parasagittal zonations within the olivocerebellar projections. I. Climbing fibers distribution in the vermis of cat cerebellum. J Comp Neurol 174:417-488.

Hallonet M, Teillet M-A, Le Douarin NM (1990) A new approach to the development of the cerebellum provided by the quail-chick marker system. Development 108:19-31.

Harkmark W (1954) Cell migrations from the rhombic lip to the inferior olive, the nucleus raphe and the pons. A morphological and experimental investigation on chick embryos. J Comp Neurol 100: 115-209.

Harkmark W (1956) The influence of the cerebellum on development and maintenance of the inferior olive and the pons. An experimental investigation in chick embryos. J Exp Zool 131:333-372.

Hekmat A, Bitter-Suermann D, Schachner M (1990) Immunocytological localization of the highly polysialylated form of the neural cell adhesion molecule during development of the murine cerebellar cortex. J Comp Neurol 291:457-467.

Künemund V, Jungalwala FB, Fischer G, Chou DKH, Keilhauer G, Schachner M (1988) The L2/HNK-1 carbohydrate of ncural cell adhesion molecules is involved in cell interactions. J Cell Biol 106: 213-223.

Le Douarin NM (1982) The neural crest (Le Douarin NM, McLaren A, eds). Cambridge: Cambridge UP.

Macklin WB, Weill CL (1985) Appearance of myelin proteins during development in the chick central nervous system. Dev Neurosci 7: 170-178.

Marin-Padilla M (1985) Neurogenesis of the climbing fibers in the human cerebellum: a Golgi study. J Comp Neurol 235:82-96.

Mason CA (1987) The development of cerebellar mossy fibers and climbing fibers: embryonic and postnatal features. In: New concepts in cerebellar neurobiology (Chan-Palay V, Palay S, eds), pp 57-88. New York: Liss.

Mason CA, Gregory E (1984) Postnatal maturation of cerebellar mossy and climbing fibers: transient expression of dual features on single axons. J Neurosci 4:1715-1735.

Mason CA, Christakos S, Catalano S (1990) Early climbing fiber interactions with Purkinje cells in the postnatal mouse cerebellum. J Comp Neurol 297:77-90.

Mugnaini E (1969) Ultrastructural studies on the cerebellar histogenesis. II. Maturation of nerve cell populations and establishment of synaptic connections in the cerebellar cortex of the chick. In: Neurobiology of cerebellar evolution and development (Llinàs $R, e d), p p$ 749-782. Chicago: American Medical Association, Education and Research Foundation Institute of Biomedical Research.

Mugnaini E, Forstronen PF (1967) Ultrastructural studies on the cerebellar histogenesis. I. Differentiation of granule cells and development of glomeruli in the chick embryo. Z Zellforsch 77:115-143.

Persohn E, Schachner M (1987) Immunoelectron microscopic localization of the neural cell adhesion molecules $\mathrm{L} 1$ aand N-CAM during postnatal development of the mouse cerebellum. J Cell Biol 105:569576.

Pourquié O, Coltey M, Thomas JL, Le Douarin NM (1990) A widely distributed antigen developmentally regulated in the nervous system. Development 109:743-752.

Rakic P, Sidman RL (1970) Histogenesis of cortical layers in human cerebellum, particularly the lamina dissecans. J Comp Neurol 139: 473-500.

Ramon y Cajal S (1911) Histologie du système nerveux de l'homme et des vertébrés. Paris: Maloine. Reprint. Madrid: Consejo Superior de Investigaciones Cientificas, 1955.

Rathjen FG, Wolff JM, Chang S, Bonhoeffer F, Raper J (1987) Neurofascin: a novel chick cell-surface glycoprotein involved in neuriteneurite interactions. Cell 51:841-849.

Schneider C, Newman RA, Asser U, Sutherland DR, Greaves MF (1982) A one step purification of membrane proteins using a high-efficiency immunomatrix. J Biol Chem 257:10766-10769.

Sotelo C, Bourrat F, Triller A (1984) Postnatal development of the inferior olivary complex in the rat. II. Topographic organization of the immature olivocerebellar projection. J Comp Neurol 222:177199.

Takagi S, Tsuji T, Kinutani M, Fujisawa H (1988) Monoclonal antibodies against species-specific antigens in the chick central nervous system: putative application as transplantation markers in chick quail chimeras. J Histochem Cytochem 37:177-184.

Tan K, Lc Douarin NM (1991) Development of the nuclei and cell migration in the medulla oblongata. Application of the quail-chick chimera system. Anat Embryol 183:321-343.

Vogt-Nilsen L (1954) The inferior olive in birds. J Comp Neurol 101: $447-481$.

Whitlock DG (1952) A neurohistological and physiological study of afferent fiber tracts and receptive areas of the avian cerebellum. $J$ Comp Neurol 97:567-635. 